\title{
Pymes: La necesidad de una nueva cultura empresarial
}

doi: 10.33264/rpa.201901-08

\author{
Galvarino Casanueva Yáñez \\ Escuela de administración UNIACC \\ Facultad de administración UNIACC
}

\section{Resumen}

El presente trabajo se basa en una parte de la tesis doctoral desarrollada por su autor, "Propuesta de valor: Un modelo de solución de negocios para las Pymes de la localidad de Melipilla - Chile", en el programa de Doctorado en Administración Gerencial de la Universidad Benito Juárez G.; y tiene por objetivo destacar la importancia de las personas en la generación de valor y la urgente necesidad actual de cambiar la cultura empresarial de las Pymes. Se justifica lo anterior, dado que los escenarios que se desenvuelven todas las empresas que conforman las denominadas Pymes (Pequeñas y Medianas Empresas) han cambiado profunda y dramáticamente $y$, hoy por hoy, todos los factores que actuaban como garantes y grandes generadores de valor para las Pymes, tales como la tecnología, el financiamiento, los mercados y la información; ya no lo son, dado que dichos factores están disponibles más que antes y, por consiguiente, al alcance de casi todas la Pymes, o por lo menos para todas las empresas que conforman la competencia de una organización económica. Efectivamente, estos factores ya no son fuentes que garanticen por sí solas la producción de ventajas competitivas, y el gran reto que enfrentan las empresas en la actualidad es lograr que sus colaboradores puedan ser más productivos y, por consiguiente, puedan crear valor. El presente artículo coloca de manifiesto que las personas o colaboradores resultan ser los grandes diferenciadores de competitividad de las empresas en la actualidad $y$, de paso, pone fin a la incertidumbre de muchos directivos sobre el verdadero valor de los colaboradores para el negocio.

Palabras claves: Pymes, creación de valor, gestión del talento, competitividad y modelos de negocio.

\begin{abstract}
The present work is based on a part of the doctoral thesis developed by its author, "Value proposal: A business solution model for SMEs in the town of Melipilla - Chile", in the Doctoral program in Management Administration of the University Benito Juárez G.; and aims to highlight the importance of people in generating value and the urgent need today to change the business culture of SMEs. This is justified, given that
\end{abstract}


the scenarios that all the companies that make up the so-called SMEs (Small and Medium Enterprises) have changed profoundly and dramatically and, today, all the factors that act as guarantors and great generators of value for SMEs, such as technology, financing, markets and information; they are no longer, given that these factors are available more than before and, therefore, within the reach of almost all SMEs, or at least for all companies that make up the competition of an economic organization. Indeed, these factors are no longer sources that guarantee the production of competitive advantages by themselves, and the great challenge facing companies today is to ensure that their employees can be more productive and, therefore, can create value. The present article shows that people or collaborators turn out to be the great differentiators of competitiveness of companies today and, in passing, puts an end to the uncertainty of many managers about the true value of employees for the business.

Keywords: SMEs, value creation, talent management, competitiveness and business models.

\section{Introducción}

Como todas las organizaciones económicas, las Pymes se enfrentan a una inmensa cantidad de desafíos, lo que provoca que muchas veces las prioridades de las empresas desplacen a un segundo plano todo lo que no signifique alcanzar las metas comerciales, siendo la selección y el reclutamiento de talentos frecuentemente postergadas. Por otro lado, es común de que no dispongan de los recursos suficientes para implementar y desarrollar su propio departamento de Recursos Humanos y, si bien algunas veces un colaborador del equipo asume dicha responsabilidad, es muy probable que no desempeñe ese rol con la misma eficiencia y eficacia que si lo hiciera una persona especializada y dedicada al cien por ciento a esta labor.

A muchas Pymes se les ha reducido el margen de gestión debido a que tienen organizaciones ineficientes e ineficaces, y otras tantas han desaparecido del mercado por la misma razón.

El gran reto que enfrentan las empresas en la actualidad es lograr que sus colaboradores puedan ser más productivos y, por lo tanto, puedan crear valor. La generación de valor es posible si la empresa logra alinear la estructura organizacional a la estrategia seleccionada, implementada y desarrollada. Lo anterior es factible si la empresa tiene una organización que requiere y no la que tiene. El presente artículo busca que las Pymes entiendan con urgencia que su área de Recursos Humanos genere portafolios de productos y/o servicios que les permita crear valor a través de los colaboradores y, además, definan cómo producirlo. Para ello se requiere además entender que el valor se mide en los resultados obtenidos por la empresa. 


\section{Desarrollo}

Las pequeñas y medianas empresas son en Chile el segmento más dinámico en cuanto a creación y muerte de empresas. El 15,2\% desaparece al primer año de vida; el mismo porcentaje dejará de existir al segundo año, y luego, al tercero morirá otro 10,4\%. Sólo el 40\% logra mantenerse al menos siete años. Estas son algunas de las cifras que reveló el documento "Dinámica Empresarial: Brechas regionales y sectoriales de las pymes en Chile”, del Ministerio de Economía, Fomento y Turismo.

El mismo estudio señala que el período más complejo que debe enfrentar una empresa son los tres primeros años desde su creación, lapso conocido como "el valle de la muerte". De acuerdo con las cifras recopiladas por el estudio, el 60\% de las microempresas logra pasar esa etapa, mientras que en el caso de las pequeñas lo hace un $80,8 \%$, en las medianas un $86,2 \%$ y en las grandes un $89,5 \%$.

Durante el trabajo de entrevistar a empresarios, directivos y/o administradores de empresas, escuchamos de las Pymes hablar acerca de sus problemáticas para crecer, crear valor agregado y encontrar colaboradores talentosos para concretar esos sueños que los llevaron a crear su unidad económica. También relataron las dificultades en la adecuación de sus empresas a los avances tecnológicos, el acceso a la información necesaria para los procesos de toma de decisiones y de la especialización requerida.

De acuerdo con Casanueva, G. (2018) "Es urgente que las Pymes puedan sortear las dificultades que actualmente se enfrentan", y que se registran con Reyes (2007) "en relación con bajos niveles de capacitación del recurso humano, limitados niveles de innovación y desarrollo tecnológico, baja penetración en mercados internacionales, acceso restringido a las fuentes de financiamiento, bajos niveles de productividad, baja capacidad de asociación y administrativa, etc."

Casanueva, G. (2018) señala “La teoría en general nos habla de la necesidad de contar con competencias distintivas (en los recursos de la organización) y con ello lograr ventajas competitivas que, entre otras cosas, durará mientras la competencia no copie el modelo, que la misma no sea tan dinámica y que la empresa poseedora de la ventaja no sea castigada socialmente por malas prácticas."

El mismo autor plantea que es necesario para las Pymes "Generar un modelo de negocios que permita crear valor con el talento. Esto permitirá a las Pymes elevar su competitividad y desarrollarse en un escenario cada vez más global y complejo." 
De acuerdo con Osterwalder et al. (2015) "Un modelo de negocio describe las bases sobre las que una empresa crea, proporciona y capta valor" Por otro lado, y de acuerdo con los mismos autores, se llama creación de valor:

Al conjunto de beneficios de la propuesta valor que se diseña para atraer a un cliente, y que esa propuesta de valor es una descripción precisa y fácil de entender e identificar por un cliente sobre lo que le estás aportando para resolver su problema. (Osterwalder et al., 2015, prólogo)

El escenario ha cambiado para todas las empresas que conforman las denominadas Pymes y, hoy por hoy, todos los factores productivos (tecnología, el financiamiento, los mercados y la información) están más fácilmente disponibles y, por esa razón, al alcance de un gran número de empresas, o por lo menos para todas ellas que conforman la competencia de una organización económica.

Anteriormente, Osterwalder \& Pigneur manifestaron que "El ámbito de la innovación en modelos de negocios está evolucionando a pasos agigantados” (2011, prólogo)

Y considerando y en concordancia con González (2005), "Resulta evidente y urgente que los recursos humanos deben ser una fuente de ventaja competitiva para cualquier empresa". (pág. xv)

El mismo autor señala: "Este modelo de soluciones de negocios exige arrancar de una definición clara de la estrategia y planes del negocio de donde debe emanar la estrategia de Recursos Humanos y donde la subordinación de ésta a aquélla debe ser evidente." (González, 2005, pág.1)

Por otro lado, García (2013) “Asimismo, se pudo observar que, en la praxis empresarial, los gerentes focalizan la generación de valor en los accionistas y clientes en primera instancia; luego, aparecen los trabajadores como un grupo de interés clave para los intereses organizacionales." (pág.23)

Resulta evidente que los colaboradores talentosos constituyen en la actualidad recursos humanos que no son transferibles, vale decir, que no lo puede tener una empresa en particular y la competencia que enfrenta a la vez. Es cierto, todas las empresas pueden generar talento, pero un colaborador talentoso específico no estará disponible para más de una empresa, a menos que la empresa que desarrolla sus funciones permita que ese colaborador talentoso abandone la organización, dado que no fue capaz de retenerlo.

El gran reto que enfrentan las empresas en la actualidad es lograr que sus colaboradores puedan ser más productivos y, en consecuencia, puedan crear valor. Ello es posible si la empresa logra alinear la estructura organizacional a la estrategia 
seleccionada, implementada y desarrollada. Lo anterior es factible si la empresa tiene una organización que requiere y no la que tiene.

A muchas Pymes se les ha reducido el margen de gestión debido a que tienen organizaciones ineficientes e ineficaces, y otros tantos han desaparecido del mercado por la misma razón.

Las Pymes deben detectar esta deficiencia organizacional a tiempo para poder adecuar estructuras idóneas a sus planes de negocios, pudiendo así reasignar su factor talento disponible en aquellos procesos que se produzcan un mayor valor, eliminando o reduciendo cargos dentro de la organización que en definitiva no producen contribución alguna, y conduciendo a la empresa a evaluar y medir con una mayor exactitud sus resultados.

Es necesario establecer una adecuada definición de la empresa, de sus planes, de su estrategia, sus objetivos y, en lo fundamental, definir y establecer indicadores de resultados que permitan evaluar constantemente el desempeño de la organización.

Posteriormente, se requiere diseñar y adoptar estructuras organizacionales convenientes y favorables para todos los procesos que requiere manejar la empresa. Para producir una condición de competitividad, la empresa debe diseñar adecuadas descripciones de cargos, con descripciones claras de su aporte a la generación de valor, de los objetivos esperados de su ejecución, de todas las actividades asociadas y sus respectivos indicadores de resultados.

Las Pymes requieren urgentemente que su área de Recursos Humanos genere portafolios de productos y/o servicios que les permita crear valor y definan cómo producirlo. Para ello se requiere entender que el valor se mide en los resultados obtenidos por la empresa.

Con ese fin, es necesario que el área de Recursos Humanos tenga pleno conocimiento de la empresa como negocio, de la estrategia formulada e implementada en la organización y que define su actuar presente y futuro, de todos los procesos involucrados, de su cartera de clientes y de sus factores diferenciales que le permiten o permitirán a la empresa producir valor.

Este conocimiento le permite al área de Recursos Humanos el insumo para capturar información e identificar las múltiples necesidades potenciales de todos los clientes internos de la organización y, con ello, establecer y construir el portafolio de productos y/o servicios de valor.

A modo de ejemplo, dar respuesta a la interrogante ¿Qué requiere el área de Operaciones del área de Recursos Humanos para poder incrementar la productividad 
de sus colaboradores? El poder entregar productos y/o servicios al área operacional, por ejemplo, convierte al área de Recursos Humanos de una organización como un socio estratégico de la empresa.

Recursos Humanos podría, por ejemplo, desarrollar un portafolio de tres a cuatro productos de alto impacto para el negocio. Por ejemplo, el producto de "Gestión del Conocimiento" para aquellas empresas que fundamentan su estrategia de negocio justamente a través de una alta gestión del conocimiento. Esto constituye un activo de alta competitividad para enfrentar y participar en el mercado. La oferta del área de Recursos Humanos será de diseñar, implementar y controlar la capitalización, distribución y aprovechamiento del conocimiento especializado que tienen las personas clave dentro de la organización. El producto generado tiene su fundamento, además, en que gran parte del valor producido por el negocio radica en el conocimiento especializado que poseen algunos de los empleados en relación con las operaciones y su administración, como sobre la identificación y, por tanto, detección de oportunidades en el mercado.

Este producto identifica sus procesos (por ejemplo, adquisición del conocimiento, explotación del conocimiento, capitalización del conocimiento, capacitación, etc.); la definición de los tipos de indicadores (por ejemplo, operativos, tácticos y/o estratégicos); su respectiva unidad (número, monetaria, porcentaje, etc.); la fórmula respectiva, y, el tipo de decisión de la empresa (por ejemplo, determinar la productividad de los procesos de toma de decisiones en tiempo y costo de las actividades de soluciones desarrolladas).

Las Pymes deben medir constantemente todos sus procesos si pretenden crecer, desarrollarse y permanecer en el mercado en forma competitiva. Una empresa que no puede o no sabe medir sus procesos y/o actividades le será imposible conocer dónde empezar siquiera para mejorar su gestión. La importancia y beneficios de la medición de los aportes de los colaboradores es clara para las organizaciones: permite el control del desempeño y facilita su corrección en favor de la productividad.

Con los indicadores definidos se puede, para el primer caso, rediseñar las cargas de trabajo de un determinado puesto, cambiar los estilos de supervisión y de dirección, apoyar los procesos de aprendizajes y de capacitación, cambiar o modificar algunas políticas empresariales, eliminar algunas prácticas improductivas dentro de la organización, entre otras. Para el segundo caso, la productividad, mediante la premiación por su concreción.

También es necesario, sin lugar a duda, mediante indicadores poder corregir aspectos relacionados con la infraestructura en que se desarrollan los procesos involucrados 
en la gestión de los colaboradores, tales como, condiciones laborales, instrumentos de trabajo, los horarios establecidos, entre otros.

Es posible concluir, que las acciones estratégicas del área de Recursos Humanos van dirigida al logro de que cada colaborador tenga y desarrolle las competencias necesarias y suficientes para contribuir en la generación de valor en la organización económica en que trabaja. Pero para que ello sea posible, su descripción de cargo debe necesariamente estar alineada a la estrategia de negocio formulada, implementada y desarrollada por la empresa.

Bajo este contexto y escenario, los colaboradores talentosos podrán:

- Obtener resultados con menos recursos

- Generar una visión de la empresa desde las responsabilidades que le asigna el puesto del trabajo a desarrollar

- Gestionar su esfuerzo mediante la innovación con la tradición

- Emplear visiones estratégicas al desarrollar su proceso de toma de decisiones

- Emplear una adecuada y eficiente dosis de autocontrol

- Lograr la adhesión de todos los involucrados en la cadena de valor; mediante la persuasión y el entusiasmo

- Desarrollar adecuados seguimientos que permiten garantizar que los procesos son ejecutados de acuerdo con lo planeado, ejerciendo eficaces controles y retroalimentando hacia los colaboradores que conforman el equipo de trabajo.

- Obtener resultados empleando las fortalezas y potencialidades de todos sus colaboradores, conformando un concepto sólido de equipo de trabajo, desarrollando inmejorables relaciones y muy confiables en toda la organización.

Generar un modelo de negocios que permita crear valor con el talento, permitirá a la Pymes elevar su competitividad y desarrollarse en un escenario global y muy complejo.

Hoy ya no se requieren planes de carrera para los colaboradores, que en última instancia fueron ideados para desarrollar una relación laboral a largo plazo. La disminución en el tiempo del ciclo de vida laboral provocó una disminución en la relación de lealtad entre empresa y colaborador, y entre otros, hace surgir la necesidad de crear una nueva relación entre la empresa y los colaboradores talentosos, en donde debe prevalecer los sistemas de oportunidades, apoyado de forma muy flexible y rentable con un sistema de competencias reales, que refleje 
resultados medibles y colocados en movimiento por propio y fuerte interés de la empresa.

\section{Conclusiones}

El escenario ha cambiado para todas las empresas que conforman las denominadas Pymes y, hoy por hoy, todos los factores (tecnología, el financiamiento, los mercados y la información) están más disponibles y, en atención a lo cual, al alcance de todos, o por lo menos para todas las empresas que conforman la competencia de una organización. Efectivamente, estos factores ya no son fuentes que garanticen por sí solas la producción de ventajas competitivas, y el gran reto que enfrentan las empresas en la actualidad es lograr que sus colaboradores puedan ser más productivos y, de tal forma que, puedan crear valor.

Por otro lado, el período más complejo que debe enfrentar una empresa son los tres primeros años desde su creación, lapso conocido como "el valle de la muerte". Para poder cruzar ese valle, y que pueda crecer y desarrollarse, las Pymes deben generar un modelo de negocios que les permita crear valor con el talento. Esto les permitirá elevar su competitividad y desarrollarse en un escenario global y complejo.

El gran reto que enfrentan las empresas en la actualidad es lograr que sus colaboradores puedan ser más productivos y, en atención a lo cual, puedan crear valor. Ello es posible si la empresa logra alinear la estructura organizacional a la estrategia seleccionada, implementada y desarrollada. Lo anterior es factible si la empresa tiene una organización que requiere y no la que tiene.

Es necesario que el área de Recursos Humanos tenga pleno conocimiento de la empresa como negocio, de la estrategia formulada e implementada en la organización y que define su actuar presente y futuro de todos los procesos involucrados, de su cartera de clientes y de sus factores diferenciales que le permiten o permitirán a la empresa producir valor.

Las acciones estratégicas del área de Recursos Humanos deben ir dirigidas al logro de que cada colaborador tenga y desarrolle las competencias necesarias y suficientes para contribuir en la generación de valor en la organización económica en que trabaja. Pero para que ello sea posible, su descripción de cargo debe necesariamente estar alineada a la estrategia de negocio formulada, implementada y desarrollada por la empresa.

Este conocimiento le permite al área de Recursos Humanos el insumo para capturar información e identificar las múltiples necesidades potenciales de todos los clientes 
internos de la organización y, con ello, establecer y construir el portafolio de productos y/o servicios que permitirán crear valor.

Las Pymes deben medir constantemente todos sus procesos si pretenden crecer, desarrollarse y permanecer en el mercado en forma competitiva. Una empresa que no puede o no sabe medir sus procesos y/o actividades le será imposible conocer dónde empezar siquiera a mejorar su gestión. La importancia y beneficios de la medición de los aportes de los colaboradores es clara para las organizaciones: permite el control del desempeño y facilita su corrección en favor de la productividad.

También es necesario, sin lugar a duda, mediante indicadores poder corregir aspectos relacionados con la infraestructura en que se desarrollan los procesos involucrados en la gestión de los colaboradores, tales como, condiciones laborales, instrumentos de trabajo, los horarios establecidos, entre otros.

Hoy en día, la función de dirigir una empresa requiere asumir e interpretar este proceso de modificación y conducir la cultura y el pensamiento de la organización empresarial en dicha dirección. La adaptación debe ser una constante en el plan de trabajo de las Pymes, y deben disponer de personas expertas y especializadas en sus roles respectivos, pero que también sean polifacéticos, capaces de llevar a cabo tareas diferentes a las suyas propias. En otras palabras, es la importancia que adquieren los colaboradores y la adecuada gestión del talento, por parte de las Pymes para la creación de valor, lo que lleva hacia una nueva cultura empresarial.

\section{Referencias}

Arellano, P. \& Jiménez, E. (2016). La estrategia competitiva: Brechas regionales y sectoriales de las pymes en Chile - periodo 2005 -2014. Recuperado del sitio del Ministerio de Economía, Fomento y Turismo: https://www.economia.gob.cl/wpcontent/uploads/2016/02/Bolet\%C3\%ADn-Din\%C3\%A1mica-Empresarial-20052014.pdf

Caballero, M., \& Freijeiro, A. (2007). Dirección estratégica de la Pyme: fundamentos y teoría para el éxito empresarial. Vigo: IdeasPropias.

Cabello, A., Reyes, R. \& Solís, P. (2007). La estrategia competitiva que adopta la empresa transnacional en el mercado global. Contaduría y administración, (220).

Casanueva, G. (2018). Propuesta de valor: Un modelo de solución de negocios para las Pyme's de la localidad de Melipilla - Chile. (Tesis Doctoral). Universidad Benito Juárez G, México.

Filion, L. J., Cisneros, L., \& Mejía, J. (2011). Administración de PYMES: Emprender, dirigir y desarrollar empresas. México: Pearson Educación. 
García, E. (2012). ¿Cómo generar valor en las empresas?. Universidad del Pacífico.

Gómez, J. (1996). Estrategia para la competitividad de las Pyme. Madrid: McGraw Hill.

González, R. (2005). Creando valor con la gente. México: Norma.

Hernández, L. (2007). Competencias esenciales y PYMEs familiares: Un modelo para el éxito empresarial. Revista de Ciencias Sociales, 13(2), 249-263.

Low, J., \& Kalafut, P. (2004). La Ventaja invisible: Cómo impulsan los intangibles el rendimiento empresarial. Empresa Activa.

Marneou, E., \& Sansores, E. (2011). El fracaso de las micro, pequeñas y medianas empresas en Quintana Roo, México: Un análisis multivariante. Revista internacional Administración \& Finanzas, 4(3). Recuperado de https://www.theibfr.com/download/riaf/2011-riaf/riaf-v4n3-2011/RIAF-V4N32011-2.pdf

Milla, A. (2002). Diez conceptos clave para entender la creación de valor. Altair Consultores SL.

Osterwalder, A., \& Papadakos, T. (2016). Diseñando la propuesta de valor: Cómo crear los productos y servicios que tus clientes están esperando. Barcelona: Deusto.

Porter, M.E. (May, 1987). From Competitive Advantage to Corporate Strategy. Harvard Business Review.

Rapallo, C. (2002). La creación de valor: una aproximación. Universidad Complutense de Madrid, Facultad de Ciencias Económicas y Empresariales.

\section{Galvarino Casanueva Yáñez}

PhD en Administración Gerencial por la Universidad Benito Juárez G.; PhD® en Administración de Empresa, Université Libre Des Science De L' Entreprise Et Des Technologies de Bruxelles; Máster en Ciencias de la Administración, Université Libre Des De L' Entreprise Et Des Technologies de Bruxelles; Master Financial Professional (AAFM); Magíster en Finanzas Corporativas por la Universidad Viña del Mar; Ingeniero Comercial (Universidad Católica del Norte; Licenciado en Ciencias de la Administración (Universidad Católica del Norte); Diplomado en Marketing Estratégico (Universidad Benito Juárez G); Diplomado en Planificación y Gestión Financiera (Universidad Benito Juárez G); Diplomado en Finanzas Corporativas (Universidad Benito Juárez G); Diplomado en Docencia Universitaria (Universidad del Pacífico). Docente en UNIACC y Asesor de Pyme's. 